\title{
GRAPHS, GROUPS AND PSEUDO-SIMILAR VERTICES
}

\author{
W. L. KOCAY \\ (Received 13 September 1982; revised 10 February 1983) \\ Communicated by W. D. Wallis
}

\begin{abstract}
Vertices $u_{0}, u_{1}, \ldots, u_{k-1}$ of a graph $X$ are mutually pseudo-similar if $X-u_{0} \cong X-u_{1} \cong \cdots \cong X-$ $u_{k-1}$, but no two of the vertices are related by an automorphism of $X$. We describe a method for constructing graphs with a set of $k \geqslant 2$ mutually pseudo-similar vertices, using a group with a special subgroup. We show that in all graphs with pseudo-similar vertices, the vertices are pseudo-similar due to the action of a group on the cosets of some subgroup.
\end{abstract}

1980 Mathematics subject classification (Amer. Math. Soc.): 05 C 25, 05 C 99.

\section{Introduction}

In Godsil and Kocay [4], a method was given for constructing graphs with a pair of pseudo-similar vertices. It was also shown that all graphs with a pair of pseudo-similar vertices could be constructed in this way. In Godsil and Kocay [5], this method was generalized somewhat, and a method was given for constructing graphs with three mutually pseudo-similar vertices. The method relied on finding a group and a subgroup with special properties.

In this paper we give a general method for constructing graphs with a set of $k \geqslant 2$ mutually pseudo-similar vertices, using an extension of the ideas of [5], based on a group with a certain subgroup. We also show that all graphs with $k$ mutually pseudo-similar vertices can be constructed in this way. In a certain sense then, we can say that graphs with pseudo-similar vertices exist because of the existence of groups with subgroups with certain properties. This more or less answers the question of why do pseudo-similar vertices exist, a question which 
was raised in Harary and Palmer [6], when investigations of the reconstruction conjecture resulted in pseudo-similar vertices being discovered. It leaves open the question of when do such groups with special subgroups exist, and how does one find them.

The main construction is based on Schreier coset diagrams, defined in Section 2 following. We refer the reader to Chapter 8 of Bollobas [1] for a concise statement of their properties.

We shall use the graph-theoretic terminology of Bondy and Murty [2].

\section{Schreier coset diagrams and partial permutations}

Let $G$ be a group generated by elements $p_{1}, p_{2}, \ldots, p_{k} \in G$. Let $p=$ $\left\{p_{1}, p_{2}, \ldots, p_{k}\right\}$, and let $K \leqslant G$ be a subgroup of $G$. Let $A=\left\{K a_{1}, K a_{2}, \ldots, K a_{n}\right\}$ be a complete set of right cosets of $K$ in $G$, where we assume that $a_{1}=e$, the identity in $G$. The Schreier coset diagram $G \bmod K$ is an edge-coloured directed graph with vertex set $A$. The edge colours are the symbols $p_{1}, p_{2}, \ldots, p_{k}$. Vertex $K a_{i}$ is joined to $K a_{i} p_{j}$ by a directed edge of colour $p_{j}$, for all $i=1,2, \ldots, n$, and all $j=1,2, \ldots, k$.

It is well known that walks in $G \bmod K$ correspond to words in the generators $P$ of $G$. Moreover, if $T$ is any spanning tree of $G \bmod K$, then the unique path in $T$ joining $K=K a_{1}$ to any vertex $K a_{i}$ defines a representative for the coset $K a_{i}$. The edges of $G \bmod K$ which are not in the spanning tree $T$ define a set of generators for $K$, as follows. If an edge $\left(K a_{i}, K a_{i} p_{j}\right)$ not in $T$ is added to $T$, this creates a unique closed walk of base $K$ in $G \bmod K$ using the edge $\left(K a_{i}, K a_{i} p_{j}\right)$ and edges from $T$. If $K a_{i}$ and $K a_{i} p_{j}$ have representatives $r_{i}$ and $r_{j}$, respectively, then the word corresponding to this closed walk is $r_{i} p_{j} r_{j}^{-1}$. As the closed walks so defined by a spanning tree $T$ generate all the closed walks of base $K$ of the graph and since every word representing an element in $K$ corresponds to a closed walk of base $K$ in $G \bmod K$ it follows that the edges of $G \bmod K$ not in $T$ define words which generate the subgroup $K$. This is the essence of the Nielson-Schreier Theorem (see Bollobas [1], Imrich [7], or Johnson [8]).

2.1. Definition. Let $X$ be a graph. Let $u, v \in V(X)$ be two vertices such that $X-u \cong X-v$, but no automorphism of $X$ maps $u$ to $v$. Then $u$ and $v$ are pseudo-similar vertices of $X$. If $u$ and $v$ are related by an automorphism, then they are similar.

Now let $X$ be a graph with a set $U=\left\{u_{0}, u_{1}, \ldots, u_{k-1}\right\} \subseteq V(X)$ of $k \geqslant 2$ mutually pseudo-similar vertices. Then $X-u_{0} \cong X-u_{1} \cong \cdots \cong X-u_{k-1}$, but 
no two of the vertices of $U$ are related by an automorphism of $X$. Many examples of such graphs for $k=2$ are given in Kocay [10]. Kimble, Stockmeyer, and Schwenk [9] given an infinite family of such graphs, one for every $k \geqslant 2$. Krishnamoorthy and Parthasarathy [14] also given an infinite family, for $k$ a power of two. Godsil and Kocay [5] and Kocay [12] give several other examples for $k=3$. Graphs with large sets of $k$ mutually pseudo-similar vertices are hard to find. We explain why this is so in Section 4.

Let $p_{i}: X-u_{i} \rightarrow X-u_{0}$ be an isomorphism, for each $i=1,2, \ldots, k-1$. Each $p_{i}$ maps $V(X)-u_{i}$ to $V(X)-u_{0}$, so that each $p_{i}$ is "almost" a permutation of $V(X)$, in the sense that only $u_{i}$ has no image under $p_{i}$, and only $u_{0}$ is not an image. We call $p_{i}$ a partial permutation of $V(X)$. For the sake of formality, we define this.

2.2. Definition. Let $V$ be any finite set. Let $p$ be a one-to-one map from a proper subset of $V$ into $V$, such that $\operatorname{Im} p \neq \operatorname{Dom} p$. Then $p$ is called a partial permutation of $V$.

Partial permuations $p$ for which $|\operatorname{Im} p|=|V|-1$ turn out to be important in the pseudo-similar vertex problem.

We shall extend each partial permutation $p_{i}$ of $V(X)$ above to a permutation $p_{i}^{*}$ of a larger set $V(Y)$. The action of the group generated by the $p_{i}^{*}, i=1,2, \ldots, k$ -1 , will then make it clear why the orginal vertices $U$ are pseudo-similar in $X$.

\section{Orbits of partial permutations}

Given the set $P=\left\{p_{1}, p_{2}, \ldots, p_{k-1}\right\}$ of partial permutations, we define a graph $\Gamma$ analogously to a Schreier coset diagram. $\Gamma$ has coloured, directed edges. The edge colours are the symbols $p_{1}, p_{2}, \ldots, p_{k-1} . V(\Gamma)=V(X)$. Vertex $u \in V(\Gamma)$ is joined by an edge of colour $p_{i}$ to $(u) p_{i}$, if $u$ has an image under $p_{i}$, for all $i=1,2, \ldots, k-1$.

Now if the $p_{i}$ were permutations, then the connected components of $\Gamma$ would be the orbits of $\langle P\rangle$, the group generated by $P$. It is easy to see that each connected component (or orbit) of $\Gamma$ would then be a Schreier coset diagram $\langle P\rangle \bmod K$, where $K$ is any point-stabiliser subgroup for that orbit. In fact, this is often used as the basis for computer algorithms which determine the order of a permutation group defined by a set of generating permutations, in terms of a nested sequence of stabiliser subgroups.

We accordingly call the connected components of $\Gamma$ the orbits of the set of partial permutations $P$. An orbit $A$ is closed if every vertex $u$ in $A$ has an in-edge 
and an out-edge of colour $p_{i}$, for every $i=1,2, \ldots, k-1$. (A loop is counted as both an in-edge and an out-edge, in case $u$ is a fixed point of $p_{i}$.) Otherwise an orbit is open.

\subsection{TheOREM. $P$ has exactly one open orbit.}

Proof. Consider $p_{i}: V(X)-u_{i} \rightarrow V(X)-u_{0} . p_{i}$ maps $u_{0}$ to $\left(u_{0}\right) p$. If $\left(u_{0}\right) p_{i} \neq$ $u_{i}$, then we can find $\left(u_{0}\right) p_{i}^{2}$, and so on. Eventually we must have $\left(u_{0}\right) p_{i}^{l}=u_{i}$, for some positive integer $l$, since $p_{i}$ is one-to-one, and only $u_{i}$ has no image under $p$. Thus there is a directed path of colour $p_{i}$ from $u_{0}$ to $u_{i}$ in $\Gamma$, for every $i=1,2, \ldots, k-1$. Thus all the $u_{i} \in U$ lie in one connected component of $\Gamma$. This is the only open orbit of $P$.

Let $\bar{U}$ denote the orbit of $P$ containing $U$, and let $V^{\prime}=V(X)-\bar{U}$.

If, $V^{\prime} \neq \varnothing$, then $p_{i} \mid V^{\prime}$, the restriction of $p_{i}$ to $V^{\prime}$, is an automorphism of the induced graph $X\left[V^{\prime}\right]$. The set $P \mid V^{\prime}=\left\{p_{i} \mid V^{\prime}\right\}$ generates a subgroup of Aut $X\left[V^{\prime}\right]$. Furthermore each connected component of $\Gamma\left[V^{\prime}\right]$ is a Schreier coset diagram for a point-stabilizer subgroup of the group of automorphisms generated by $P \mid V^{\prime}$

We can make the open orbit $\bar{U}$ into a closed orbit, thereby completing the partial permutations, as follows.

Let $G$ denote the free group (see Johnson [8]) generated by the symbols of $P=\left\{p_{1}, p_{2}, \ldots, p_{k-1}\right\}$. Let $T$ denote a spanning tree of $\Gamma[\bar{U}]$. We shall define a subgroup $K$ of $G$ as follows. Label the vertex $u_{0}$ in $\Gamma[\bar{U}]$ by the coset $K e$, where $e$ is the identity in $G$. For each vertex $u \in \bar{U}$, label $u$ with $K w(u)$, where $w(u)$ is the unique word in the $p_{i}$ corresponding to the unique path in $T$ from $u_{0}$ to $u$. For each directed edge $u v$ of colour $p_{i}$ in $\Gamma[\bar{U}]$ define the label of $u v$ as $w(u) p_{i} w(v)^{-1}$. Let $K$ be the subgroup of $G$ generated by the labels of the edges of $\Gamma[\bar{U}]$. Using Schreier's method (see Bollobas [1]), we have:

3.2. THEOREM. The Schreier coset diagram $G \bmod K$ contains $\Gamma[\bar{U}]$ as an induced subgraph.

This gives the following theorem.

3.3. THEOREM. Let $X$ be a graph with a set $U=\left\{u_{0}, u_{1}, \ldots, u_{k-1}\right\}$ of $k$ mutually pseudo-similar vertices. Let $p_{i}: X-u_{i} \rightarrow X-u_{0}$ be isomorphisms, for $i=$ $1,2, \ldots, k-1$. Then $X$ can be extended to a graph $Y$, and each $p_{i}$ can be extended to a permutation $p_{i}^{*}$ of $V(Y)$ such that:

(i) $X$ is an induced subgraph of $Y$;

(ii) each $p_{i}^{*}$ is an automorphism of $Y$;

(iii) the vertices of $U$ are all similar in $Y$;

(iv) the vertices of $V(Y)-V(X)$ are all in the same orbit of Aut $Y$ as $U$. 
Proof. Let $G$ and $K$ be as above. Let $A=\left\{K a_{t} \mid i \in I\right\}$ be a complete set of right cosets of $K$ in $G$. Let $V(Y)=V^{\prime} \cup A$. Notice that $V(X) \subseteq V(Y)$ since $V^{\prime} \subseteq V(X)$, and $\bar{U} \subseteq A$. We define the edge set of $Y$ as follows.

If $u v \in E(X)$, where $u, v \in V^{\prime}$, then $u v \in E(Y)$.

Let $u_{0}$ be joined to vertices $M$ of $V^{\prime}$ in $X$. Then join each coset $K a \in V(Y)$, where $a$ is a word in the $p_{i}$, to $(M) a$. This is possible, since $p_{i} \mid V^{\prime}$ is an automorphism of $X\left[V^{\prime}\right]$. Thus arbitrary words in the $p_{i}$ define permutations of $V^{\prime}$. If the coset $K a$ is in $V(X)$, this agrees with edges already present in $X$, since each $p_{i}$ is an isomorphism from $X-u_{i}$ to $X-u_{0}$, that is, it adds no new edges to $X$. Moreover, this includes all edges from $V^{\prime}$ to $\bar{U}$ in $X$.

We have left only edges in $X[\bar{U}]$. Let $u v$ be an edge of $X$ in $X[\bar{U}]$. Suppose that $u$ and $v$ correspond in $V(Y)$ to cosets $K a_{1}$ and $K a_{2}$ of $K$. For each word $a \in G$, define an edge $\left(\mathrm{Ka}_{1} a, \mathrm{Ka}_{2} a\right)$ in $Y$. Clearly this includes all edges of $X[\bar{U}]$. It adds no new edges to $X$, since each $p_{i}$ is an isomorphism, and since the only circuits in the Schreier coset diagram $G$ mod $K$ are contained in $\Gamma[\bar{U}]$, by the definition of $K$.

It follows that $X$ is an induced subgraph of $Y$. Moreover each $p_{i}$ extends to a permutation $p_{i}^{*}$ in the permutation representation of $G$ in terms of the right cosets of $K$. Thus each $p_{i}^{*}$ is an automorphism of $Y$. Since $\left(u_{0}\right) p_{i}^{l_{i}}=u_{i}$, for each $i=1,2, \ldots, k-1$, where $l_{i}$ is a positive integer, it follows that all the vertices of $U$ are similar in $Y$, and clearly all the new vertices added to $X$ to get $Y$ lie in one orbit, the orbit of $\bar{U}$.

Theorem 3.3 is the $k$-analog of the main theorem of Godsil and Kocay [4] for pairs of pseudo-similar vertices. The main difference is that in [4] the group $G$ was made finite, so that the resultant graph $Y$ was finite. In Theorem 3.3 above, the graph $Y$ is always infinite.

If we adjoin relators to $G$, consistent with Aut $X\left[V^{\prime}\right]$, to make $G$ into a finite group, then $K$ will become finite, too. So long as the cosets of $K$ corresponding to the vertices of $\Gamma[\bar{U}]$ are all distinct, and $G \bmod K$ does not add any new edges to $\Gamma[\bar{U}]$, then we can use this finite $G$ and $K$ to create a finite graph $Y$. If $X[\bar{U}]$ contains no edges, then this will work fine. But if $X[\bar{U}]$ contains edges, then we cannot be sure that a finite $G$ will not add new edges to $X$.

3.4. Problem. Do there exist graphs $X$ with $k \geqslant 3$ mutually pseudo-similar vertices for which the graph $Y$ cannot be made finite?

3.5. Problem. Do there exist graphs $X$ with $k \geqslant 3$ mutually pseudo-similar vertices for which $V^{\prime}=\varnothing$ ? 
In [4] (see also [11]), it was proved that when $k=2$, we must always have $\left|V^{\prime}\right| \geqslant 6$. All known examples of graphs with $k \geqslant 3$ have $V \neq \varnothing$. It would seem reasonable to expect that when $k \geqslant 3$, we can find graphs $X$ with $V^{\prime}=\varnothing$.

In the same way as we defined the orbits of the set $P$ of partial permuations, we can define the orbits of $P$ on the pairs of vertices of $X$, that is, the pair-orbits of $P$. Unlike Theorem 3.1, $P$ will in general have several open pair orbits. A solution to Problems 3.4 and 3.5 would apparently require a study of the pair-orbits of $P$.

\section{Constructing graphs with $k$ mutually pseudo-similar vertices}

In [5] a method was given for constructing graphs with three mutually pseudosimilar vertices, based on a group $G$ with a special subgroup $K$. Section 3 above shows that corresponding to any graph with $k$ mutually pseudo-similar vertices, we can construct a group $G$ with a subgroup $K$, such that the vertices are pseudo-similar due to the action of $G$ on the cosets of $K$.

In this section we show how to use these ideas to construct graphs with pseudo-similar vertices.

4.1. Example. Let $k=2$. Let $U=\left\{u_{0}, u_{1}\right\}$ and let $p: X-u_{1} \rightarrow X-u_{0}$. Then $\Gamma[\bar{U}]$ is easily determined. It must be a directed path of length $l \geqslant 1$ from $u_{0}$ to $u_{1}$. When $k=2$, we must have $\left|V^{\prime}\right| \geqslant 6$ (see [4] or [11]).

Since $\Gamma[\bar{U}]$ is a tree, we must have $T=\Gamma[\bar{U}]$. It follows that $G$ is the free group generated by $p, G=\langle p\rangle$, and $K$ is the identity. If we make $G$ finite it becomes a cyclic group, and we then easily construct pseudo-similar vertices.

Only in the case of $k=2$ can we completely classify all groups $G$ giving rise to pseudo-similar vertices, that is, all those which contain an element of period three or more.

When $k \geqslant 3$, it is impossible to classify, in general, all the possible equivalence classes for the coset diagram $\Gamma[\bar{U}]$, where we define equivalence of two coset diagrams $\Gamma_{1}[\bar{U}]$ and $\Gamma_{2}[\bar{U}]$ as follows.

If $U=\left\{u_{0}, u_{1}, \ldots, u_{k-1}\right\}$ and $P=\left\{p_{1}, p_{2}, \ldots, p_{k-1}\right\}$, defined $p_{i j}=p_{i} p_{j}^{-1}$, for $1 \leqslant i<j \leqslant k-1$, where the product of isomorphisms is read from left to right. Then $p_{i j}: X-u_{i} \rightarrow X-u_{j}$. Let $P^{\prime}=P \cup\left\{p_{i j} \mid 1 \leqslant i<j \leqslant k-1\right\}$.

For each $p_{i j} \in P^{\prime}$, we add edges of colour $p_{i j}$ to $\Gamma[\bar{U}]$, in the same way as edges corresponding to $p_{i}, i=1,2, \ldots, k-1$, were introduced. This results in the completed coset diagram $\Gamma^{\prime}[\bar{U}]$.

4.2. Definition. Two coset diagrams $\Gamma_{1}[\bar{U}]$ and $\Gamma_{2}[\bar{U}]$ with sets of partial permutations $P_{1}$ and $P_{2}$, respectively, are equivalent if there exist two bijections, 
both denoted $\theta$, between the vertex-sets, and edge-sets of $\Gamma_{1}^{\prime}[\bar{U}]$ and $\Gamma_{2}^{\prime}[\bar{U}]$ such that:

(i) for each colour $p \in P_{1}^{\prime}, \theta$ maps the edges of colour $p$ in $\Gamma_{1}^{\prime}[\bar{U}]$ to the edges of some colour $p^{\prime} \in P_{2}^{\prime}$ in $\Gamma_{2}^{\prime}[\bar{U}]$;

(ii) for each colour $p \in P_{1}^{\prime}, \theta$ either preserves the direction of all the edges coloured $p$, or reverses the direction of all of them.

4.3. ExAMPLE. Let $k=3$. Let $U=\left\{u_{0}, u_{1}, u_{2}\right\}$ and let $p_{1}: X-u_{1} \rightarrow X-u_{0}$ and $p_{2}: X-u_{2} \rightarrow X-u_{0}$. Then $p_{12}=p_{1} p_{2}^{-1}: X-u_{1} \rightarrow X-u_{2}$, and $P^{\prime}=$ $\left\{p_{1}, p_{2}, p_{12}\right\}$. If $|\bar{U}|=3$, so that $\bar{U}=U$, there are exactly two possible equivalence classes for $\Gamma[\bar{U}]$, shown in Figures 4.1 and 4.2.

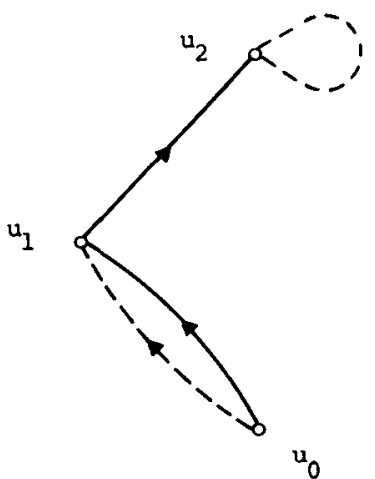

$\Gamma_{1}[\overline{\mathrm{U}}]$

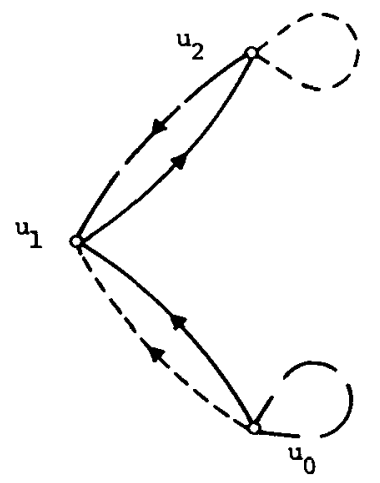

$\Gamma_{1}[\bar{U}]$

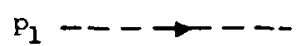

$\mathbf{P}_{2}$

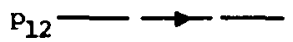

Figure 4.1. Type I

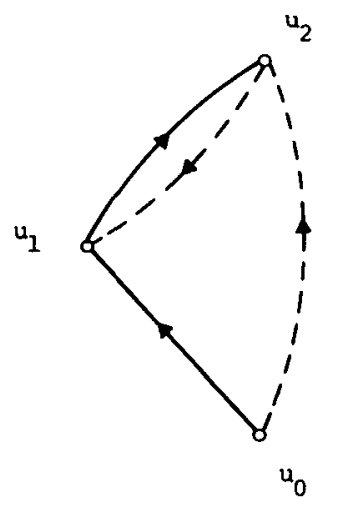

$\Gamma_{2}[\overrightarrow{\mathrm{U}}]$

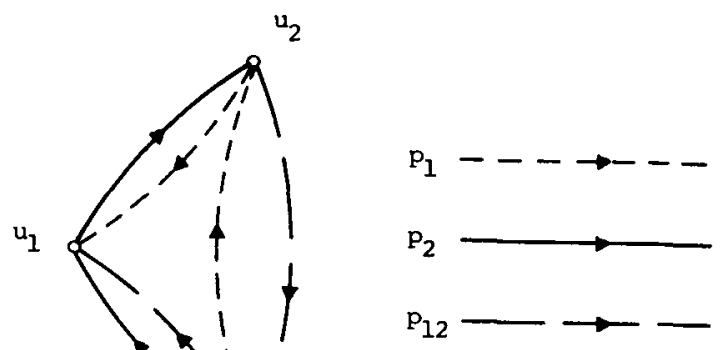

$\mathbf{u}_{0}$

$\Gamma_{2}^{\prime}[\overline{\mathrm{U}}]$

Figure 4.2. Type II 
Both of these configurations have been used in [5] to construct graphs with three mutually pseudo-similar vertices. If we choose the solid edges in each coset diagram as the spanning tree $T$, then we get the following subgroups $K$.

I. $K=\left\langle p_{1} p_{2}^{-1}, p_{2}^{2} p_{1} p_{2}^{-2}\right\rangle$.

II. $K=\left\langle p_{1} p_{2}^{-2}, p_{2}^{2} p_{1} p_{2}^{-1}\right\rangle$.

If $k=3$ and $|\bar{U}|=4$, there are exactly 8 equivalence classes for $\Gamma[\bar{U}]$. If $k=4$ and $|\bar{U}|=4$, there are exactly 19 equivalence classes for $\Gamma[\bar{U}]$. These are enumerated in Kocay [13].

A basic recipe for constructing a graph $X$ with $k$ mutually pseudo-similar vertices can then be given as follows. We first need the definition of a Cayley graph.

4.4. Definirion. Let $G$ be a group with a generating set $H$ such that $e \notin H$. The Cayley graph $C(G, H)$ has vertex set $G$ and edge set $\{(g, h g) \mid g \in G$, $h \in H\}$. Thus $G$ acts as a group of automorphisms of $C(G, H)$, by right multiplication. In case $C(G, H)$ has no other automorphisms, $C(G, H)$ is called a graphical regular representation, or GRR, of $G$ (see Godsil [3]). Except for 10 exceptional graphs of order at most 32, all finite, non-abelian groups which are not generalized dicyclic groups have GRR's (see [3]).

\subsection{Construction.}

1. Given $k$, let $U=\left\{u_{0}, u_{1}, \ldots, u_{k-1}\right\}$.

2. Choose a set $\bar{U}$ of vertices so that $U \subseteq \bar{U}$.

3. Given colours $p_{1}, p_{2}, \ldots, p_{k-1}$, draw a directed path of colour $p_{i}$ from $u_{0}$ to $u_{i}$ in $\bar{U}$, for $i=1,2, \ldots, k-1$.

4. If there are any vertices of $\bar{U}$ not on the directed path from $u_{0}$ to $u_{i}$ of colour $p_{i}$, then add edges of colour $p_{i}$ so that a disjoint union of cycles is induced on these vertices by the edges of colour $p_{i}$.

5. Choose a spanning tree $T$ of the resultant $\Gamma[\bar{U}]$, and label the vertices of $\Gamma[\bar{U}]$ with the coset representatives of $K$, given by the unique path in $T$ from $u_{0}$ to each vertex.

6. Read off a set of generators for $K$.

7. Find a finite group $G$ generated by $P=\left\{p_{1}, p_{2}, \ldots, p_{k-1}\right\}$ and identify $K$ as a subgroup.

8. Draw a Cayley graph for $G$, and call it $X\left[V^{\prime}\right]$.

9. Find the pair-orbits or $P$ on $\bar{U}$. Make some of the pair-orbits into edges in $X$, if desired.

The resultant graph $X$ satisfies $X-u_{0} \cong X-u_{1} \cong \cdots \cong X-u_{k-1}$, since $G$ acts on the right cosets of $K$ in $X\left[V^{\prime}\right]$ by right multiplication. If $X\left[V^{\prime}\right]$ is a GRR, then we can be sure there will be no automorphisms of $X$ connecting any of the 
$u_{i} \in U$. For then the only automorphisms of $X\left[V^{\prime}\right]$ are those arising from $G$. It follows that the vertices $u_{i}, i=0,1, \ldots, k-1$, are mutually pseudo-similar in $X$.

The reason graphs with $k \geqslant 4$ mutually pseudo-similar vertices are hard to find is because of the difficulty with step 7 , namely finding a suitable group $G$ and identifying the subgroup $K$. The author knows of no general algorithm for finding such a $G$ and $K$.

\section{References}

[1] B. Bollobas, Graph theory (Springer-Verlag, New York, 1979).

[2] J. A. Bondy and U. S. R. Murty, Graph theory with applications (Macmillan, London, 1976).

[3] C. D. Godsil, Graphs with regular groups (Ph.D. Thesis, University of Melbourne, 1979).

[4] C. D. Godsil and W. L. Kocay, 'Constructing graphs with pairs of pseudo-similar vertices', $J$. Combinatorial Theory Ser. B, to appear.

[5] C. D. Godsil and W. L. Kocay, 'Graphs with 3 mutually pseudo-similar vertices', submitted for publication.

[6] F. Harary and E. Palmer, 'On similar points of a graph', J. Math. Mech. 15 (1966), 623-630.

[7] W. Imrich, 'Subgroup theorems and graphs', Combinatorial mathematics V, (Springer-Verlag, New York, 1977).

[8] D. M. Johnson, Topics in the theory of group presentations (Cambridge University Press, 1980).

[9] R. Kimble, P. Stockmeyer and A. Schwenk, 'Pseudosimilar vertices in a graph', J. Graph Theory 5 (1981), 171-181.

[10] W. L. Kocay, 'On pseudo-similar vertices', Ars Combinatoria 10 (1980), 147-163.

[11] W. L. Kocay, 'Some new methods in reconstruction theory', Proceedings of the Ninth Australian Combinatorial Conference (Springer, to appear).

[12] W. L. Kocay, 'A small graph with three mutually pseudo-similar vertices', in preparation.

[13] W. L. Kocay, 'An enumeration of coset diagrams for pseudo-similar vertices', in preparation.

[14] V. Krishnamoorthy and S. Parthasarathy, 'Cospectral graphs and digraphs with given automorphism group', J. Combinatorial Theory Ser. B 19 (1975), 204-213.

Department of Computer Science

University of Queensland

St. Lucia, Queensland

Australia
Department of Computer Science

University of Manitoba

Winnipeg, Manitoba

Canada R3T 2N2 